\title{
B01 The Seismic Method: Using Acoustic Pressure to Estimate Reservoir Geometry, Fluid Proporties and Pressure Changes
}

Martin Landrø, NTNU

The role of seismic data in hydrocarbon production has gradually increased over the last three decades. First, the introduction of 3D seismic data resulted in a significant improvement of the imaging of a given reservoir. More precise volume estimates and better reservoir management could be attributed to the $3 \mathrm{D}$ seismic method. The next gradual step was the dawn of seismic inversion techniques combined with rock physics. The combination of the two disciplines leads to a significant improvement in reservoir characterization. A nice feature of seismic inversion techniques is that it is easier to assess uncertainty estimates to the estimated reservoir parameters. Uncertainty is however, still an important challenge in seismic reservoir characterization.

The third major step is the growth of time lapse seismic studies. Several successful 4D onshore studies were reported in the mid-90ies, but this did not lead to a major step change. However, in the North Sea, this activity bloomed after the commercial success of the Gullfaks 4D study in 1995-1997. This success lead to a rapid growth in the number of 4D studies in the North Sea. Today, time-lapse seismic is a well established tool, and future challenges are quantitative methods, resolving production changes within thin layers and to increase the accuracy of the method so that it can be used for stiffer reservoir rocks like for instance carbonates.

Another key challenge for the seismic method is to estimate pore pressure changes. Results so far indicate that it is easier to detect a pore pressure increase than a pore pressure decrease from time lapse seismic data.

Presently, there is a weak link between well test data and seismic data. For 4D interpretation, pressure measurements from wells are used as important information to constrain the interpretation. However, there are few examples where a more direct link between 4D and well test data has been demonstrated. This is definitely a future challenge: How should we combine well test data with 4D data in an efficient way? It is well known that reservoir heterogeneities like a fault close to the well impacts the pressure behaviour in the well, so there is obviously a direct link between well test measurements and detailed interpretation of faults from 3D seismic data. In a 4D seismic setting this link can be further improved, since the 4D seismic can distinguish between low and high permeable zones within the reservoir, which again could be coupled to well test measurements. 